Abstract

\title{
Psychological Health, Quality of Life, and Physical Activity Levels of Caregivers of People with Dementia ${ }^{+}$
}

\author{
Anna Chatzaki 1,*, Costas Chryssanthopoulos ${ }^{1}$, Maria Maridaki ${ }^{2}$, Michael Koutsilieris ${ }^{1}$ and \\ Anastassios Philippou ${ }^{1}$ \\ 1 Physiology Laboratory, Medical School, National and Kapodistrian University of Athens, \\ 11527 Athens, Greece \\ 2 School of Physical Education and Sport Science, National and Kapodistrian University of Athens, \\ 17237 Athens, Greece \\ * Correspondence: anna.may.1992@hotmail.com \\ + Presented at the 9th Greek Conference of Biochemistry and Physiology of Exercise, Thessaloniki, Greece, \\ 18-20 October 2019.
}

Published: 2 September 2019

\begin{abstract}
AIM: Sedentary lifestyle is related to many non-communicable diseases and psychological disorders. Family caregivers often have physical and psychological health problems, similar to those characterizing a sedentary lifestyle. The aim of this study was to determine the levels of physical activity, depression, anxiety, burden, and quality of life in Greek family caregivers of people with dementia and in non-caregiving controls, and to explore whether physical activity can affect their psychological health and quality of life. MATERIAL \& METHOD: A cross-sectional survey was conducted including 159 volunteers, 100 family caregivers of people suffering from dementia, and 59 age- and gender-matched non-caregivers. Six questionnaires were provided to the caregivers and five to the non-caregivers, including a demographic questionnaire, a stress Scale-BAI, a depression scale-BDI, a quality of life scale (WHOQOL-BREF), a physical activity Questionnaire-RPAQ, and the scale of burden-ZBI, which was given only to caregivers. The two groups were compared in order to be revealed whether psychological and quality of life variables are related to or are independent of care. RESULTS: Caregivers appear to have a worse quality of life $(p<0.001)$ and lower levels of physical activity $(p<0.05)$ compared to age- and gender-matched non-caregivers. No significant differences were found between groups regarding anxiety and depression $(p>0.05)$. Interestingly, the less active caregivers and non-caregivers have a worse quality of life than the more active individuals $(p<0.05)$, while, particularly, the less active caregivers exhibited higher levels of depression compared with the more active caregivers $(p<$ 0.05). On the other hand, it was found that higher levels of PA associate with lower levels of depression $\left(r_{\mathrm{P}}=-0.2, p<0.05\right)$ and quality of life becomes better $\left(r_{\mathrm{P}}=0.303, p<0.001\right)$. Moreover, it was revealed that stress and depression levels, quality of life, and burden (caregivers) are correlated with each other in both groups $(p<0.01)$. CONCLUSIONS: Greek caregivers of people with dementia exhibited lower levels of physical activity and a worse quality of life than non-caregivers, while physical activity appeared to improve quality of life and depression in both groups.
\end{abstract}

Keywords: caregivers; dementia; physical activity

(C) 2019 by the authors. Licensee MDPI, Basel, Switzerland. This article is an open access article distributed under the terms and conditions of the Creative Commons Attribution (CC BY) license (http://creativecommons.org/licenses/by/4.0/). 